\title{
A Multichannel Information System to Build and Deliver Rich User-Experiences in Exhibits and Museums
}

\author{
Antonio Gentile ${ }^{1,2}$, Salvatore Andolina ${ }^{1,2}$, Antonio Massara ${ }^{1}$, Dario Pirrone ${ }^{1,2}$, \\ Giuseppe Russo ${ }^{1}$, Antonella Santangelo ${ }^{1}$, Eleonora Trumello ${ }^{1}$, Salvatore Sorce ${ }^{1,2}$ \\ ${ }^{1}$ InformAmuse s.r.1. \\ Viale delle Scienze Ed 16 - 90128 Palermo Italy \\ \{gentile, andolina, massara, pirrone, russo, santangelo, trumello, sorce\}@informamuse.com \\ ${ }^{2}$ Dipartimento di Ingegneria Chimica, Gestionale, Informatica e Meccanica \\ Università degli Studi di Palermo- Viale delle Scienze Ed 6 - 90128 Palermo Italy \\ gentile@unipa.it, \{andolina,dpirrone,sorce\}@dinfo.unipa.it
}

\begin{abstract}
In this article a multichannel information system to build and deliver rich user experiences in exhibits and museums is presented. The system was designed to use information about a particular exhibit or museum while delivering a wide user experience based on different distribution channels. The overall information is used to build different solutions that can be delivered simultaneously on different media from touch-screen installations to portable devices like smartphones. Moreover, all the devices signed in the environment are able to communicate to each others to increase the level of the usability of the system. A case study and analysis of experimental results are also provided.
\end{abstract}

Multichannel Information System, User-experience, musuems, exhibition, personalization, integration

\section{INTRODUCTION}

The definition of an information system involves different research aspects and methodology choices that have to be sustained. The most important aspect to stress is the interaction process between the users and the system. A good organization of the contents to propose is a good starting point to build effective information systems although the central point of the problem still is the model of the interaction process. Usually, this interaction is described as the user experience and involves all the previously defined aspects of the process. The definition of a proper user experience starts from the definition of the typical users for the information system. A common way to define the users is to define a set of parameters and, accordingly to a proper configuration, a set of classes to model typical categories. This classification process is often performed off-line and as a prerequisite of the information system. Many classifications have been proposed. Falks [1] proposes five different categories of users: Explorers, Facilitators, Experience Seekers, Professional/Hobbyist, and Rechargers. The personal context of the user includes aspects such as prior knowledge and specific interests, the physical context of the exhibit and the socio-cultural context related to inter and between group interactions.
As stated, the model definition is the prerequisite to build a system able to personalize the contents for a specific user. In [2] a simple taxonomy of the possible models is presented. User's models can be organized in an implicit way or explicitly built with auto-evaluation tests administered to the users.

In the definition of complex information systems for museums and exhibits three main research areas have been observed:

- Contents organization: this is mostly a knowledge definition problem and can be approached in several ways.

- User modelling: the definition of a user model is an open research field and involves several aspects from the ontological model of the user to the localization and tracking problems in an indoor environment

- Contents fruition and user experience definition: this category of problems is strictly related to human computer interaction field.

This paper addresses many of the aspects presented in this introduction with the solutions adopted in the development of the QROUTEME system. QROUTEME is a complex, multichannel information system to build and deliver rich user-experiences in museums and exhibits.

The system organization includes contents personalization and delivery in different types of media. We use both stationary (totem/kiosk) media handlers based on surface computing to allow users a simple gesture-based interaction process, and mobile (smartphones, tablet) ones to allow adaptation in contents fruition. The purpose is to build a more personalized user experience. We define adaptivity as the process where information related to user context are used to adjust the presentation of information and the user representation. This is a key feature for systems that have limitations related to technical resources such as screen sizes, battery consumption, ergonomics, connectivity and limitations related to the user interaction process like haptic 
capabilities, working memory or even simply limited amount of time for a visit.

The aspect of contents organization related to adaptation has been observed in several related studies. A first important classification is presented in [3] where essentially three types of adaptive strategies are described. The first, defined as "adapted strategy", induces pre-optimization of contents and resources from the awareness of limitations. The second type is an "adaptive strategy", where the system reacts to external changes in a sort of parameterized way and "adapting strategies" where is possible to handle different strategies according to environmental inputs. The adopted solutions for the QRouteMe system are essentially of the second type. We were aware of a series of environmental limitations and produced a set of strategies related to each of the initial constraints. A typical example is the visualization of information in smartphones with different screens. The produced output is able to adjust the contents organization according to screen size without any additional processing. Another important feature in terms of adaptation is related to positioning process. In particular, the system is able to determine the user's position inside an indoor environment by means of fiduciary posts or interaction at kiosk location. In fact, current smartphones have a resolution for indoor environments that is no better than 10 meters and that is clearly inadequate in many situations, such as fairs and museum exhibits. From an infrastructural point of view an important achievement for our system is related to his lowlevel deployment cost. The produced system doesn't need an expensive infrastructure to produce a rich user experience. The infrastructure is organized with one of more servers to organize information and communication between the users and the system, a set of stationary media serving points connected to servers to show information and a wireless network to allow users' mobile devices to exchange information. To locate users no other sensor/actuator devices are needed.

Our focus is on interaction and utilization of new technologies, such as surface computing used to develop more intuitive gesture-based interfaces. In addition, the increased computing capacity of current portable devices shifts some of the computational load to the client side of the system. Another important aspect is the possibility to constantly interact with everyday life devices that can collect large amount of information to be used in personalized systems. Also the integration between devices with different connection capabilities is a technological key aspect to explore.

The rest of the paper is arranged as follows: next section reports related works and technological issues. The third section of the paper presents the system infrastructure. The fourth section presents an implemented use case and the related experimental results. The paper ends with conclusions and future works.

\section{RELATED WORKS}

The definition and implementation of complex systems able to support users in indoor environments like an exhibit or a museum is an active and multidisciplinary research field. The overall problem has been approached from many perspectives starting from the process of contents definition and organization to personalization from different users. In addition to this, many related problems have been investigated such as the localization for indoor environments.

An early work is the definition of a set of different prototypes both for indoor and outdoor guides called Cyberguide [4] that was designed as a combination of four main components: a cartographer component including the map (or maps) of the physical environments that the tourist is visiting, a librarian component which is the information repository containing all the information to be presented, a navigator component used to keep track of the users' positions in the environment and a messenger component used to record message exchanges to/from users to system.

Other relevant systems are the Hippie/HIPS project [5] that is focused on development of an exhibition guide, providing guidance and information services. From the observations about the visitor's movements through the exhibition the systems creates a user profile and suggests other interesting exhibits or paths inside the current exhibits. The TellMaris [6] system is an example of a mobile tourist guide developed combining both two and three-dimensional graphics running in a mobile phone.

The possibility to automatically define related information for a guide has been exploited in many projects such as the PEACH project [7] where the generation of some position related contents and post-visit reports are automatically performed. The CHIP project [8] tries to combine Semantic Web techniques to provide personalized access to digital museum collections both online and in the physical museum. Most of the works are focused on an explicit definition of a knowledge base while some works tries to implicitly define a user model. The user model definition is based on statistical models rather than recommendation techniques [9].

Another point of view to build a museum guide is to target not just a single user but also a group visiting a museum. The Sotto voce [10] system is designed specifically with this goal providing a communication mechanism to support interaction.

From an architectural organization a complex system able to produce and adapt contents for different media has to be organized as a client server architecture or as a multiagent system. The drawbacks of the two approaches are well known. The server machine is obviously a point of failure and also the communication through the network can be a critical point while an explicit message passing mechanism has to be implemented for an agent based system together with a knowledge base used to define the communication ontology for the agents.

According to users' localization there are essentially two types of location technologies: the indoor positioning and the outdoor positioning. The second class of problems has been solved using satellite infrastructure with GPS (Global Positioning System). The reached level of accuracy is in the order of some meters and is generally a good accuracy for outdoor-based information applications. The indoor location suffers of the degrading reception of GPS-based systems. 
Furthermore the accuracy is not so important while in many cases is more important a system able to recognize boundaries and positioning a person through a symbolic location (e.g. "in the main hall" or in "the first room").

Several methods have been proposed to solve the problem using different media like infrared beacons [11] [12] or radio signals from wireless LAN [13] [14], RFID technology [15] or cameras and microphones [16] to detect user location. One of the main drawbacks of the proposed approach is related to the initial cost to organize a large-scale event like an exhibit.

All the proposed methods require an electronic infrastructure to facilitate measurements with all the necessary sensor/actuator devices. In this case an approach able to give to users not continuous information of their position but discrete information can be a good compromise in a costs/benefits tradeoff.

We are looking to provide an inexpensive, building-wide infrastructure to be used in a large-scale type of events with a number of users that can be measured in thousands of people. So the utilization of a fiduciary marker able to be easily recognized from users is a natural solution for this type of problems. In this way the user localization shifts from a continuous to a discrete problem. Some similar approaches have been recently used to solve this problem. In [17] a system used as a location-based conference guide called Signpost is presented. The system works only with Windows Mobile phones but is able to be used in large-scale events with no further costs due to other equipment.

Another way to achieve the same functionality is through the detection of the position by comparison between a set of floorplans and an image taken from the cell-phone camera [18]. This method has a major disadvantage because it requires all the floorplans for a particular building.

\section{SYSTEM INFRASTRUCTURE}

The main goal of the QROUTEME system is to provide users with domain information, according to their actual need and to the context they are currently part of.

To this end, we decided to design the system infrastructure according to the client-server paradigm. This choice allowed us to obtain an easy-to-implement, easy-toscale and easy-to-manage framework of components, which can be suitably used to reach our intended goal.

The QROUTEME infrastructure is composed of three main components:

- QRouteMe Platform;

- QRouteMe Front End;

- QRouteMe On Site.

The first one operates at the server side, whereas the second and third components operate at the client side.

Figure 1 shows the three main components, their modules and the overall data flow among them.

In the following sub-sections we will give a short overview of each component.

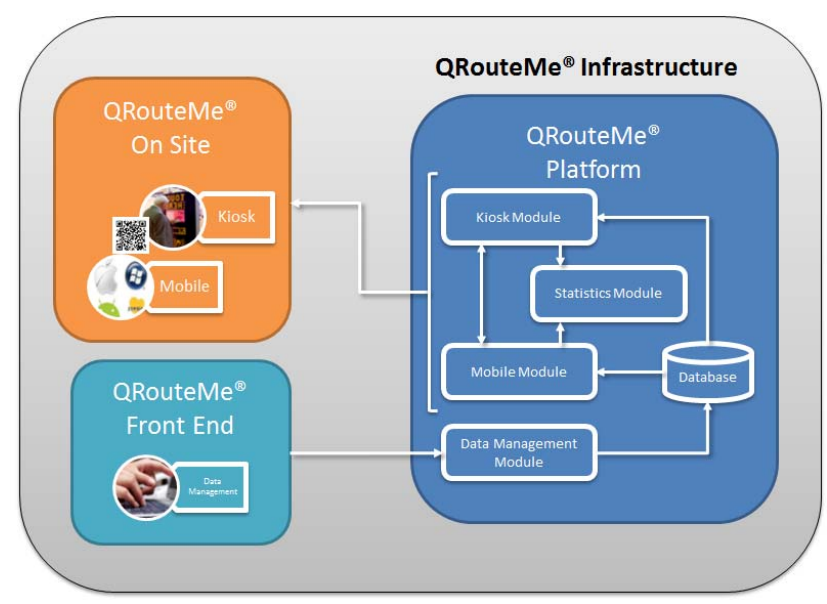

Figure 1. QROUTEME® Infrastructure data flow diagram.

\section{A. QRouteMe Platform}

On the server side, the QROUTEME Platform component main activities are data management, data processing and data storage. This component carries out most of the processing tasks of the whole system, and it is composed by four different modules (see Figure 1):

- Kiosk;

- Mobile;

- Statistics;

- Data Management.

All of them interact, whether directly or not, with a Database based on the relational model. Its schema has been defined in order to be easily adapted to the changing data domains and to the kind of service users have to be provided with.

The data set views are generated using a server side scripting language, so they can be suitably and quickly adapted to the customer requirements in terms of data presentation.

The database can also be populated by the client himself, using the QROUTEME Front End component. This feature gives the customer the possibility to keep the system up-todate at any time, and to keep the full control on data integrity and correctness (these are critical properties, especially at the deployment time).

The Kiosk and the Mobile modules manage the users' requests according to the device they are actually using. These modules extract the useful data from the database, and compose the consequent information taking into account which channel the user is currently using to interact with the system.

The Statistics module traces the users as they surf the information whilst completely preserving their anonymity and protecting their privacy.

By monitoring a great number of parameters, it can provide administrators with a wide range of useful information about the user behavior. Customers may know how many users accessed the application, which and how many pages they were viewing, and so on, according to their feedback needs. This way, they could fine tune the 
interaction to improve the final user experience, or simply they could evaluate the system effectiveness.

Of course, the information provided by the module can go through different levels of detail. It can carry on the overall system evaluation, the separate kiosk and mobile evaluation, or the evaluation of each device involved in the interaction.

The Data Management module handles the interaction between the Platform component and the applications running on the Front End component.

\section{B. QRouteMe Front End}

The QROUTEME Front End component consists of adhoc tools made available to the customer and implemented according to his needs.

The main goal of these tools is to allow the customer to create, read, update and delete data, either directly or by giving data owners the possibility to do it by themselves.

This component interacts directly with the Data Management module within the Platform component (see Figure 1). This ensures that all its activities can be carried out by keeping data integrity and correctness, while avoiding possible conflicts.

Furthermore this component improve the system adaptability to the application domain changes, as well as the preservation of data privacy, making possible to avoid the intervention of personnel not related to the customer (such as technicians).

\section{QRouteMe On Site}

The QROUTEME On Site component provide the end users with the information suitably composed by the Platform component (actually by its Kiosk and Mobile modules, see figure 1). It represents the system interface with the users.

This component consists of a set of applications which can be made to work together in different ways according to the customer needs. The QROUTEME On Site component allows people to access the available information by means of kiosks suitably configured, or by their personal mobile devices.

Concerning the mobile devices in particular, applications can be natively designed and implemented for the most common operating systems (Android, iOS, Windows Mobile and Symbian), thus exploiting all software and hardware features. Nevertheless, if there is no need to use specific hardware features (such as accelerometers, cameras, positioning systems), a cross-platform web-based solution can be used, in order to have a better portability.

This component also links both kiosk- and mobile-based information access ways by means of QR codes (Quick Response) [19]. People can search for information on a kiosk and then transfer the desired output on their mobile, or people can directly access pieces of information by shooting at QR codes, provided that their mobile device is equipped with a $Q R$ reader.

\section{CASE STUDY}

\section{A. Deployment event}

QROUTEME has been implemented for the first time at the "Vinitaly 2011", the largest fair dedicated to the Italian wine industry, which takes place every year in Verona (Italy).

Our customer was the Istituto Regionale della Vite e del Vino (IRVV, Sicilian Institute of Vine and Wine), who asked us to implement an information system inside the "Sicilia" pavilion within the fair. The main goals of the system, as requested by IRVV, were:

- allow visitors to access information about Sicilian wine producers, even in mobility;

- $\quad$ help them moving around the pavilion;

- obtain a report to evaluate what services visitors usually look for.

\section{B. QRouteMe layout at Vinitaly 2011}

After the study of the location and of the booth layout, we decided that the optimal deployment of our system could be as follows:

- 14 fixed touch totems (InformaPoint);

- 2 web and database servers;

- 1 wired LAN (designed by us and implemented by Verona Fiere);

- 1 wireless LAN with controlled access (designed by us and implemented by Verona Fiere);

- 1 database to store producers data, the booth layout, and other additional data;

- 1 app for mobile users of iOS-based devices (iPhone, iPod, iPad);

- web based presentation of information for mobile users with other operating systems;

- $234 \mathrm{QR}$ codes for the quick access to contextualized information.

Figure 2 shows the position of the fourteen touch-screen totems in the pavilion. The kiosk position was decided based on surface area and previous event knowledge about visitor entrance patterns. 


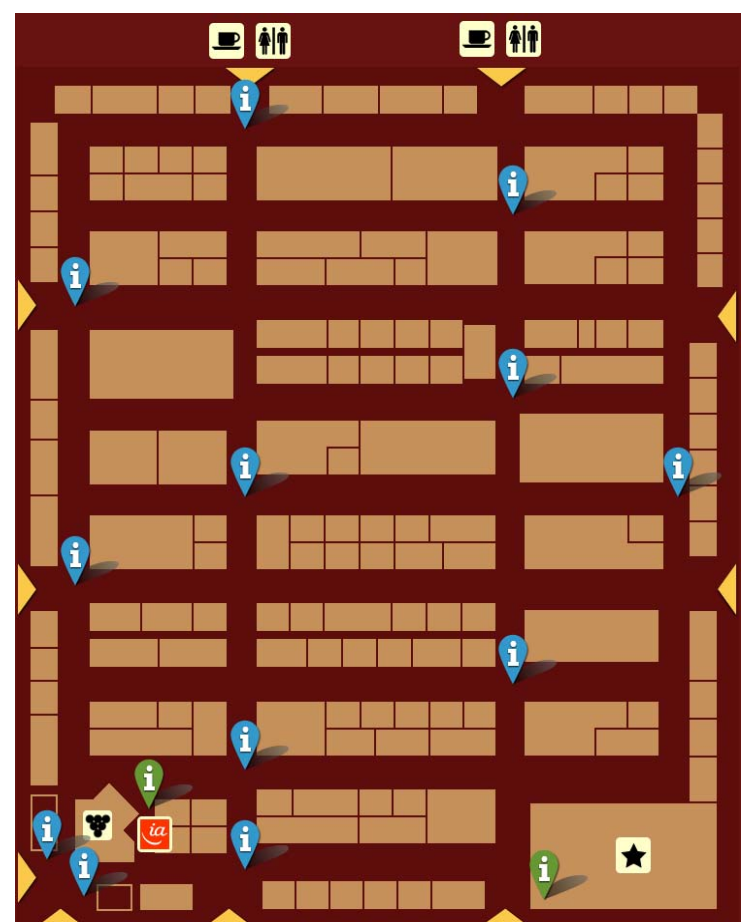

Figure 2. Kiosks layout within the pavillion

The yellow triangles in figure 2 represent the ways of access to the pavilion, whereas the blue and green placeholders show the points where the fourteen touch totems InformaPoint were placed.

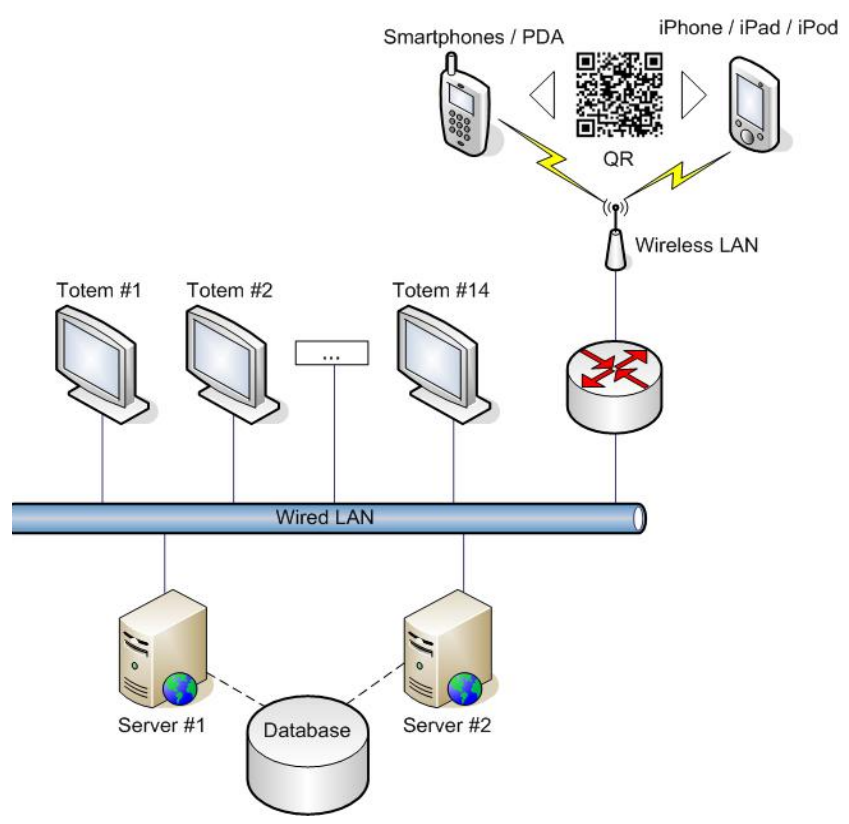

Figure 3. QROUTEME installation setup
Kiosk covered an average surface area of about $500 \mathrm{~m}^{2}$, with higher concentration around the main entrance (at the lower left corner) and along the left side, closest to neighboring pavilions, where they could be easily accessed by visitors upon entering the pavilion. 3.

The overview of the installation setup is shown in figure

\section{Information provided by QRouteME}

According to the IRVV needs and requirements, the QROUTEME system deployed at Vinitaly 2011 provided visitors with information about:

- producers list, with alphabetical and direct search features, and related details:

○ producer information;

$\circ$ wine of the year;

- list of produced wines;

○ QR code to quick access producer's information;

- producer booth position within the pavilion.

- wine areas and related details:

$\circ$ area description and pictures;

$\circ$ producers belonging to a given vine area.

- information about the IRVV;

- information about services available within the pavilion;

- information about the system.

Visitors equipped with a smartphone could access the system by means of their devices, exploiting the selfpositioning feature based on the use of QR codes. They could see their current position within the pavilion, could access directly to information about the producer they were looking at, and could see the shortest path to reach a given producer's booth from their current position.

Besides this, users of iOS-based devices could download an app (http://itunes.apple.com/it/app/sicilia-vinitaly2011/id427870112? $\mathrm{mt}=8$ ) that allow them to navigate a 3D model of the pavilion, to contact a producer via phone or email, and to share their own experience on the most common social networks.

\section{Interaction with QRouteMe}

Interfacing with the totem touch-screen, the visitors of "Vinitaly 2011" could read on screen any information about exhibitors, their wines and position that each had in the Sicily hall. The system also provides the opportunity to obtain a print screen of the shortest path to go, from the totem queried by users, to booth of the desired exhibitor. In addition to this, the information view of every exhibitor contain a QR code, through which the user could easily download on his smartphone a map of the route computed from the system.

All data necessary for the operation of the fourteen InformaPoints (text, video and images) are stored into the two server machines of QROUTEME, in which was implemented a relational database. Moreover, the 
QROUTEME system includes a website designed specifically for viewing on mobile devices, in order to allow an adequate fruition of the information present in QROUTEME. All URLs, encoded using the QR code, link to this web site. To give an immediate access to these web pages, each exhibitor was provided with a print of his QR codes, that link to the pages containing the information about the grower. In fact, shooting a QR code it is possible to navigate to a web page without having to type the URL, work very uncomfortable using mobile devices.

Particular attention was paid to the users of the iPhone devices, through the development of ad-hoc application (Figure 4). This way, it was possible to organize optimally display and navigation of information according to the Apple device hardware specifications.

This application includes a software module capable of decoding QR codes and interpret them correctly, according to the type of information that they contain. In addition to that, was developed a module capable of locating the visitor without using geo-referencing systems. In order to identify its location, the system asks the user to photograph a QR code. The decoded string indicates uniquely the position that photographed QR takes on the map (Figure 4), which coincides with that of the visitor who stands before it. Furthermore, this position can be used by the visitor as a starting point for generating a path to another point of interest.

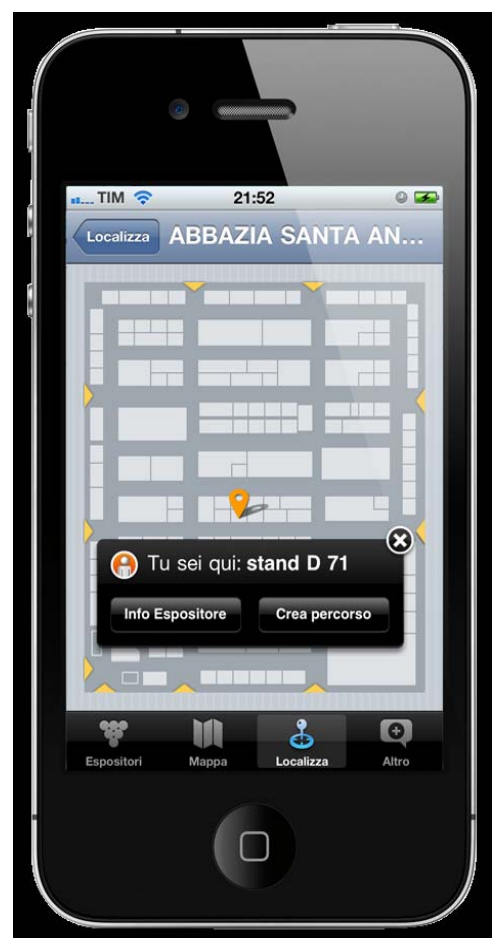

Figure 4. iOS app for Vinitaly 2011

\section{E. System Usage Reports}

QROUTEME has been developed to allow friendly and intuitive interaction with the user. To this end the fourteen InformaPoints were equipped with a GUI designed only for touch-based interaction that proved to be very effective, and easily understood by users.

The QROUTEME system was deployed at the "Vinitaly 2011" international wine fair, held in Verona (Italy) from 7 to 11 april 2011. It worked for five days, during opening hours (ten hours a day).

This proved to be a good test bed for our system, both for its effectiveness and for its robustness against any kind of human or technical fault.

The whole fair counted 8 big pavilions of about $\sim 8,000$ $\mathrm{m}^{2}$ each $(\sim 72,000 \mathrm{sq}$. $\mathrm{ft}$. $)$, with a total count of more than 150.000 visitors from all over the world, either business operators or tourists. The system was actually deployed inside the "Sicilia" pavilion, one of the largest pavilions within the fair.

During the working hours, we observed the behavior of people while interacting with totems, taking into account comments and observations.

At the first day of the fair, we observed that the main goal of people was to single out a given wine producer from the available alphabetical list. This task was at first carried out by means of a vertical scrolling bar, with the aid of an alphabetical index allowing for the fast scrolling to the corresponding letter. Despite this possibility, we saw that that task was not so easy to accomplish, mainly due to the length of the list (more than 220 producers).

We then decided to test the modularity of our system, by adding an interaction way while the system was up and running. In fact, we set up the on-screen keyboard direct search feature actually modifying the Kiosk module within the Platform component (see Figure 1 for reference), with no need to interrupt the service operation.

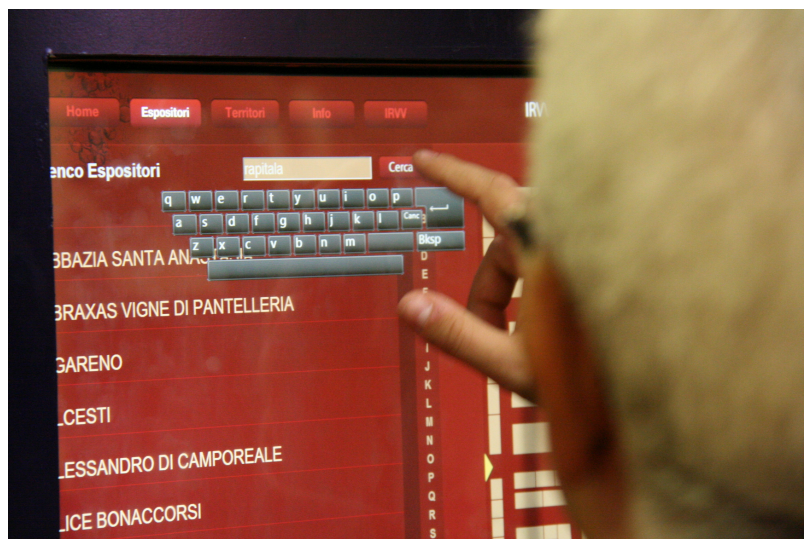

Figure 5. Keyboard on-screen for a faster search

Once deployed, the direct search by means of the superimposed on-screen keyboard became one of the features people found most useful during their interaction with totems (Figure 5). This feature allowed them to easily 
find their preferred producer, its location within the pavilion, the path to reach it from the current position and the list of produced wines.

During our survey, we observed that users experienced no other particular difficulty while interacting with the system. Once facing a totem, users started to interact with it in a few touches, independently of age.

Starting from the second fair day, we observed growing queues of people in front of totems, thus confirming the effectiveness of the interaction model and of the totem layout within the pavilion.

Our informal observations about QROUTEME usage by InformaPoint totems were confirmed by the final reports generated by the system, with more than 40.000 accesses to the pages, and more than 13.000 accesses to the producers' pages. Table I shows a short resume of those reports both for the Italian and English version of pages.

TABLE I. QROUTEME USAGE REPORT (TOTEMS)

\begin{tabular}{|r|r|r|r|r|}
\hline \multicolumn{1}{|c|}{ Date } & \multicolumn{2}{|c|}{ Single page access } & \multicolumn{2}{c|}{ Producer pages } \\
\hline & \multicolumn{1}{|c|}{ ITA } & \multicolumn{1}{c|}{ ENG } & ITA & \multicolumn{1}{c|}{ ENG } \\
\hline $7 / 4 / 2011$ & 4987 & 688 & 1480 & 180 \\
\hline $8 / 4 / 2011$ & 8019 & 1063 & 2876 & 366 \\
\hline $9 / 4 / 2011$ & 11469 & 945 & 4091 & 307 \\
\hline $10 / 4 / 2011$ & 8379 & 529 & 2793 & 187 \\
\hline $11 / 4 / 2011$ & 4146 & 122 & 1402 & 42 \\
\hline Subtotal & 37000 & 3347 & 12642 & 1082 \\
\hline Total & & 40347 & \multicolumn{3}{c}{13724} \\
\hline
\end{tabular}

Concerning the interaction from mobile devices, of course we could not observe the behavior of people while using their devices to access the system. We can only shortly discuss the reports resumed in Table II.

TABLE II. QROUTEME USAGE REPORT (MOBILES)

\begin{tabular}{|r|r|}
\hline \multicolumn{1}{|c|}{ Data } & Single page access \\
\hline $7 / 4 / 2011$ & 813 \\
\hline $8 / 4 / 2011$ & 810 \\
\hline $9 / 4 / 2011$ & 626 \\
\hline $10 / 4 / 2011$ & 427 \\
\hline $11 / 4 / 2011$ & 172 \\
\hline Total & 2848 \\
\hline
\end{tabular}

The number of total accesses to single pages is low if compared to that obtained by totem (with a ratio of about one to fifteen), due to different reasons:

- totems were well placed all around the pavilion, so that users could easily see them;

- totem places were clearly indicated with big banners hanging from the pavilion ceiling;

- there were no equivalently visible signs about the possibility to access the system by means of mobile devices;

people who knew about the mobile access found anyway easier to use totems, instead of registering to the wireless network, download the app, install it, and finally use it.
For those users who did access the system via mobile devices (and available to fill a short satisfaction report), the level of satisfaction was rather high, as despite a small overhead, the app offered more contextualized information, practically "out of your pocket", quoting one of those users.

\section{CONCLUSIONS AND FUTURE WORKS}

In this works a multichannel information system able to build and deliver rich user experiences in exhibits and museums was proposed. The system infrastructure and a complete instance, deployed in a large exhibit, were presented. The system presents several advantages starting from low deployment costs for very large environments. This is the result of a shifting paradigm: the user localization is approached as a discrete problem using a set of fiduciary markers that are able to auto-locate the users. The system architecture is built as a modular set of components that are chosen and selectively deployed according to the specific needs of the application, without any loss of effectiveness.

Current works aims at implementing customizable frontend for final customers to allow for complete contents organizations and easiness of user experience.

\section{REFERENCES}

[1] Falk, J.H.: "Identity and the Museum Visitor Experience". Left Coast Press (2009)

[2] Tsvi Kuflik, Judy Kay and Bob Kummerfeld, "Lifelong Personalized Museum Experiences" Proc. User Modeling, Adaptation and Personalization (UMAP 2010) pp. 9-17

[3] Wasinger, R., Kruger, A., Jacobs, O.: Integrating intra and extra gestures into a mobile and multimodal shopping assistant. In: 3rd International Conference on Pervasive Computing. (2005) 297-314

[4] Long, S., Aust, D., Abowd, G., Atkeson, C.: "Cyberguide: Prototyping context-aware mobile applications". Conference on Human Factors in Computing Systems, pp. 293-294. Vancouver, Canada (1996)

[5] R. Oppermann and M. Specht. "A Context-Sensitive Nomadic Exhibition Guide". In Second Symposium on Handheld and Ubiquitous Computing ( HUC2K), pages 127- 149, 2000.

[6] C. Kray, K. Laakso, C. Elting and V. Coors. "Presenting route instructions on mobile devices". In Proceedings of the International Conference on Intelligent User Interfaces 2003 (IUI 03), pages 117124,2003

[7] Stock, O,. Zancanaro, M., Busetta, P., Callaway, C., Krüger, A., Kruppa, M.,Kuflik, T., Not E. and Rocchi, C. (2007) "Adaptive, Intelligent Presentation of Information for the Museum Visitor in PEACH. User modeling and User Adapted Interaction”. 17(3), pp 257-304

[8] Aroyo, L., Stash, N., Wang, Y., Gorgels, P., Rutledge, L.: "CHIP demonstrator: Semantics-driven recommendations and museum tour generation". In: Proc. of the Sixth Intl. Semantic Web Conf. (ISWC07). (2007) 879-886

[9] Albrecht, D.W., Zukerman, I.: "Special issue on statistical and probabilistic methods for user modeling". User Modeling and UserAdapted Interaction 17(1-2) (2007)

[10] Aoki, P.M., Grinter, R.E., Hurst, A., Szymanski, M.H., Thornton, J.D., Woodruff, A.: Sotto voce: exploring the interplay of conversation and mobile audio spaces. In: CHI '02: Proceedings of the SIGCHI conference on Human factors in computing systems, New York, NY, USA, ACM Press (2002) 431-438

[11] Butz, A., Baus, J., Kruger, A.: “Augmenting buildings with infrared information". In: Proceedings of the International Symposium on 
Augmented Reality (ISAR), IEEE Computer Society Press (2000)M. Young, The Technical Writer's Handbook. Mill Valley, CA: University Science, 1989.

[12] Harter, A., Hopper, A.: "A distributed location system for the active office". IEEE Network 8(1) (1994) 62-70

[13] Bahl, P., Padmanabhan, V.: Radar: "An in-building rf-based location and tracking system". In: IEEE INFOCOM 2000. (2000)

[14] Krumm,J., Cermak,G., Horvitz,E.: "Rightspot: A novel sense of location for a smart personal object". In: Proceedings of Ubicomp 2003. (2003) 36-43

[15] Brandherm, B., Schwartz, T.: "Geo referenced dynamic bayesian networks for user position- ing on mobile systems". In: Proceedings of the International Workshop on Location- and Context-Awareness (LoCA). LNCS 3479, Springer (2005)
[16] Xuehai, B., Abowd, G., Rehg, J.: "Using sound source localization in a home environment". In: Proceedings of Pervasive Computing 05, Springer (2005)

[17] Alessandro Mulloni, Daniel Wagner, Istvan Barakonyi, Dieter Schmalstieg, "Indoor Positioning and Navigation with Camera Phones", IEEE Pervasive Computing, vol. 8, no. 2, pp. 22-31, Apr.June 2009, doi:10.1109/MPRV.2009.30

[18] Hile, H.; Borriello, G.; , "Positioning and Orientation in Indoor Environments Using Camera Phones" Computer Graphics and Applications, IEEE , vol.28, no.4, pp.32-39, July-Aug. 2008 doi: 10.1109/MCG.2008.80

[19] About 2D Code / QR Code.com, Denso-Wave, http://www.densowave.com/qrcode/aboutqr-e.html, Retrieved 18 june 2011 